\title{
An Rate Adjustment Algorithm for Scalable URLLC in Beyond 5G Networks
}

\author{
Chanho Yoon, Seokki Kim, *Nurul Huda Mahmood, *Irfan Muhammad, Gweondo Jo, and Young-Jo Ko \\ Future Mobile Communication Research Division Research Lab. \\ ETRI, Daejeon 34129, Republic of Korea \\ chyoon@etri.re.kr \\ *Centre for Wireless Communications \\ University of Oulu, Findand \\ nurulhuda.mahmood@oulu.fi
}

\begin{abstract}
Ultra-reliable low latency communications (URLLC) service class introduced in Fifth Generation (5G) New Radio (NR) caters to mission-critical applications with stringent quality of service requirements. Meeting such demanding design goals require a paradigm shift in resource management procedures. Conventional reactive schemes need to be replaced by novel and proactive resource management schemes that can efficiently meet such demanding design targets. In this paper, we propose a novel location-aware transmission rate adjustment scheduling procedure for URLLC networks based on a predictive interference management scheme. Prior to scheduling, a geographical relocation of some of the receivers, which cannot overcome a minimum reliability/outage requirement set by physical layer, is enforced if the corresponding long term channel and interference statistics fail to meet a proposed criteria. Extensive system level simulations show that the proposed relocation and interference prediction based scheduling method meets the reliability constraint and enhances utilization factor of the scalable URLLC network resource significantly. The proposed scheme demonstrates around an order of magnitude lower outage probability compared to a baseline conventional scheme, while having a higher resource efficiency.
\end{abstract}

Index Terms - Rate adaptation, resource management, scalable URLLC, URLLC

\section{INTRODUCTION}

The fifth generation (5G) New Radio (NR) wireless system is expected to evolve/support various specialized services in practical scenarios in vertical domains such as factory automation, automotive controls, e-health care, smart metering, Tactile Internet, etc. Such machine type communication (MTC) aspect will play a significant role in future beyond $5 \mathrm{G}$ networks [1]. Among the various MTC services, missioncritical MTC can be realized by ultra-reliable low-latency communications (URLLC) service class introduced in 5G. URLLC includes applications with the requirements of ultrareliable and extreme low-latency to enable real-time control and automation of dynamic process in the industrial field and various other verticals. For example, URLLC application designed for factory automation in the beyond $5 \mathrm{G}$ era faces stringent requirements suggested by codeword block error rate (BLER) of $10^{-9}$ and sub-millisecond latency [2]-[5].

In order to facilitate URLLC services in MTC networks, there are critical challenging points that physical as well as resource management layer must overcome. These include uncertainty in the traffic, random channel variation due to large and small scale fading (i.e., fluctuation in received signal to interference noise ratio), and random interference caused by other cell scheduling [4]. In order to overcome these challenges and enable URLLC, 5G NR adopted to modify the numerology by introducing short transmission time intervals (TTI) as low latency enabler [6] and allocate more than sufficient wireless resource in the physical layer to ensure ultra reliability [7]. However, this may be practiced in limited scenarios, where the transmission rate is nearly fixed and the demand for network capacity is far below the inherent network potential. Future beyond 5G systems will need to serve URLLC applications with nodes requiring higher network capacity while the stringent requirements are still in demand. Thus, URLLC network with scalability requirements will need to address additional challenges in the radio resource management (RRM) sector.

Previously, predictive resource efficient RRM algorithm for scalable URLLC was investigated [8][9]. The predicted interference variations are accurate enough to set minimal margin for achieving both reliability and network resource efficiency. However, the simulation environment for evaluation and analysis was focused on a single receiver in a single serving cell with multiple interference sources. Thus, there is an initiative to evaluate further whether the schedulers in a multiple serving cells scenario can independently function for achieving the URLLC constraints by collecting interference reports from their serving mobile stations (MSs). This paper aims to extend/evaluate the previous findings from the interference model of discrete state space discrete-time Markov chain to a 3D channel model environment consisting of multiple receiver's interference measurement affecting the serving cell's resource management scheduling.

In this paper, we propose a novel transmission rate adjustment scheduling procedure for URLLC networks based on the predictive interference management algorithm. Prior to scheduling, a geographical relocation of some receivers, which cannot overcome a minimum reliability/outage requirement set by the physical layer, is enforced if the corresponding long term channel and interference statistics fail to meet a proposed 


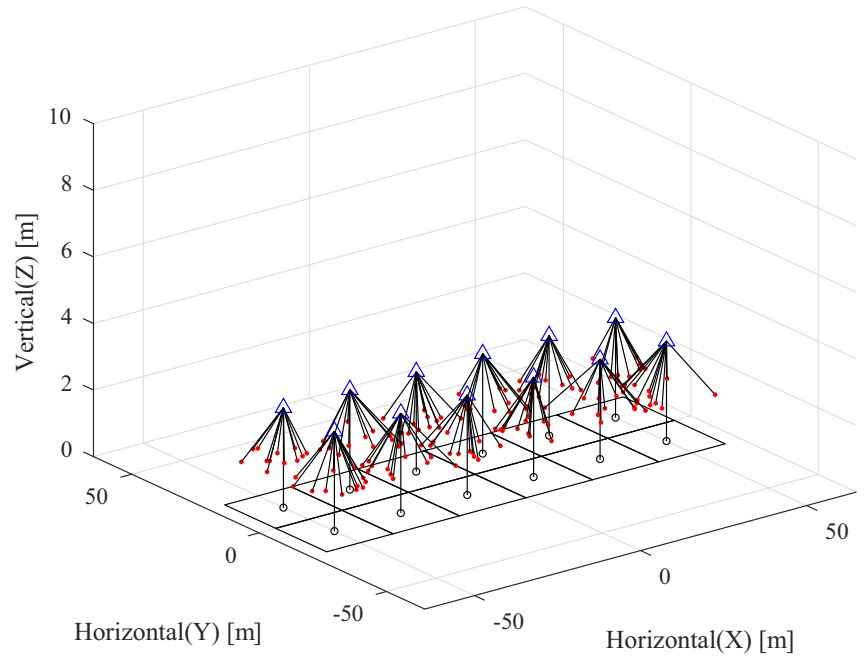

Fig. 1. URLLC base and mobile station indoor deployment scenario model

criteria. As evaluated in [10], severe interference is present near the cell edge boundaries when multi-cell is operated such that a frequency partitioning scheme is suggested as a solution for fulfilling the stringent URLLC requirements. We propose that if service for MS near the cell boundaries can be relocated, frequency reuse- 1 factor could be achieved. If service is still required at an original location, frequency reuse-1/2 or cell layout should be redesigned. However, frequency partitioning leads to inefficiency since the total available bandwidth is reduced. System level simulations show that the proposed relocation and interference prediction based scheduling method meets the reliability constraint and enhances utilization factor of the scalable URLLC network resource significantly.

\section{SySTEM MOdel}

In this section, we first overview the deployment scenario and the proposed resource allocation rules of the system model for multi-cell multi receivers. The system is based on the indoor office scenario of multi-cell defined in 3GPP TR 38.901 [11]. As shown in Fig. 1, there are 12 cells each equipped with their own antenna panels in geographically separated sites. The network operates without any coordination among other base stations (BSs). Each MS is camped on a single cell and is served with single-user multiple-input and multiple-output (SU-MIMO) rather than multi-user MIMO (MU-MIMO) due to ultra-reliability requirements. Each antenna port is mapped to antenna elements within antenna panels via analog beamforming, and multiple antenna ports are used for digital beamforming or spatial diversity/multiplexing. The 3GPP NR 3D channel model [11] is assumed for the downlink, where large scale fading and fast fading is generated based on horizontal and vertical angle of departure and arrivals with respect to antenna models. Perfect uplink signal reception is assumed for interference reports from MSs.

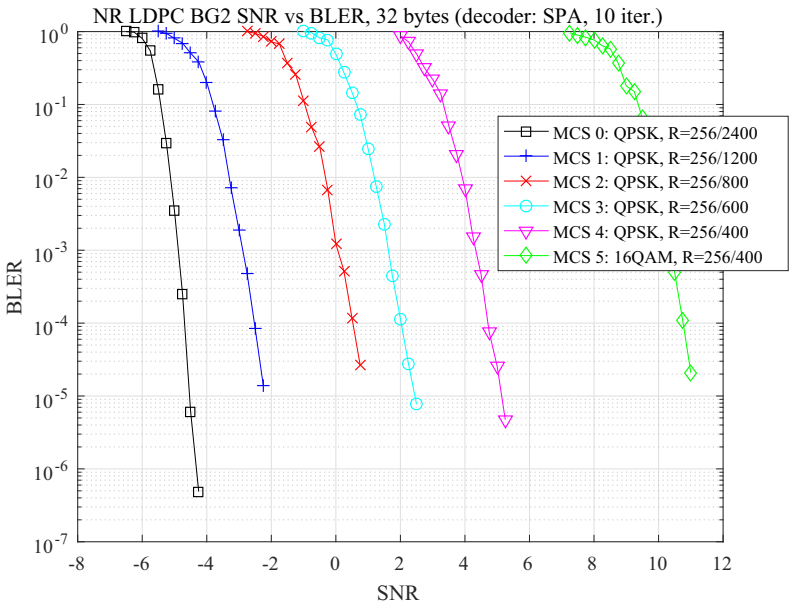

Fig. 2. BLER reference curves with arbitrary MCS sets for URLLC system level simulation

\section{A. Simple physical resource allocation system model}

The BS transmits a fixed data of size $D$ with channel coding redundancy to combat random bit errors. Let us assume a bandwidth of $20 \mathrm{MHz}$ and mini-slot duration of 4 OFDM symbols with $60 \mathrm{kHz}$ subcarrier spacing are given as a physical resource. This corresponds to an extremely lowlatency transmission slot of approximately $71 \mu \mathrm{s}$. Then, total resource element (RE) of 1200 is obtained if 300 out of 512 subcarriers (512 FFT size) in the frequency domain are utilized for data transmission. Fig. 2 shows BLER curves for some arbitrary modulation and coding set (MCS) levels for system level simulation. The MCS BLER results are reference curves for translating transmission error probability at a receiver's given signal to interference noise ratio (SINR) in the system model. As shown in Table 1, the lowest MCS level 0 is the case when all of the single mini-slot resource is used solely for a single MS receiving $D=256$ information bit. In other words, only one MS at a mini-slot time is served.

Our proposed novel scheduling scheme works as follows. First, the BS considers the received interference of the MS with the worst received SINR within the group of MSs that are to be scheduled. It is assumed that the BS has perfect knowledge of the interference status of the serving cell MSs. If this interference is measured to be low, the BS schedules all the MSs with higher level MCSs, thus allowing more MSs to be served within a single mini-slot. For example, if an MS with the worst received SINR within a selected group obtains SINR of $3 \mathrm{~dB}$, we can observe from Table I that the users can be scheduled with MCS 3 . Hence, 4 MSs can be concurrently served in a slot duration.

Since downlink transmission data rate is essentially represented by MCS, the scheduler must choose the right MCS and amount of physical REs to fulfill the reliability and latency constraints, in consideration of the receiver's predicted SINR. To ensure the stringent quality of service, the received signal of MCS level should be able to overcome fading and interference caused by other BS in the downlink. Thus, choosing the right 

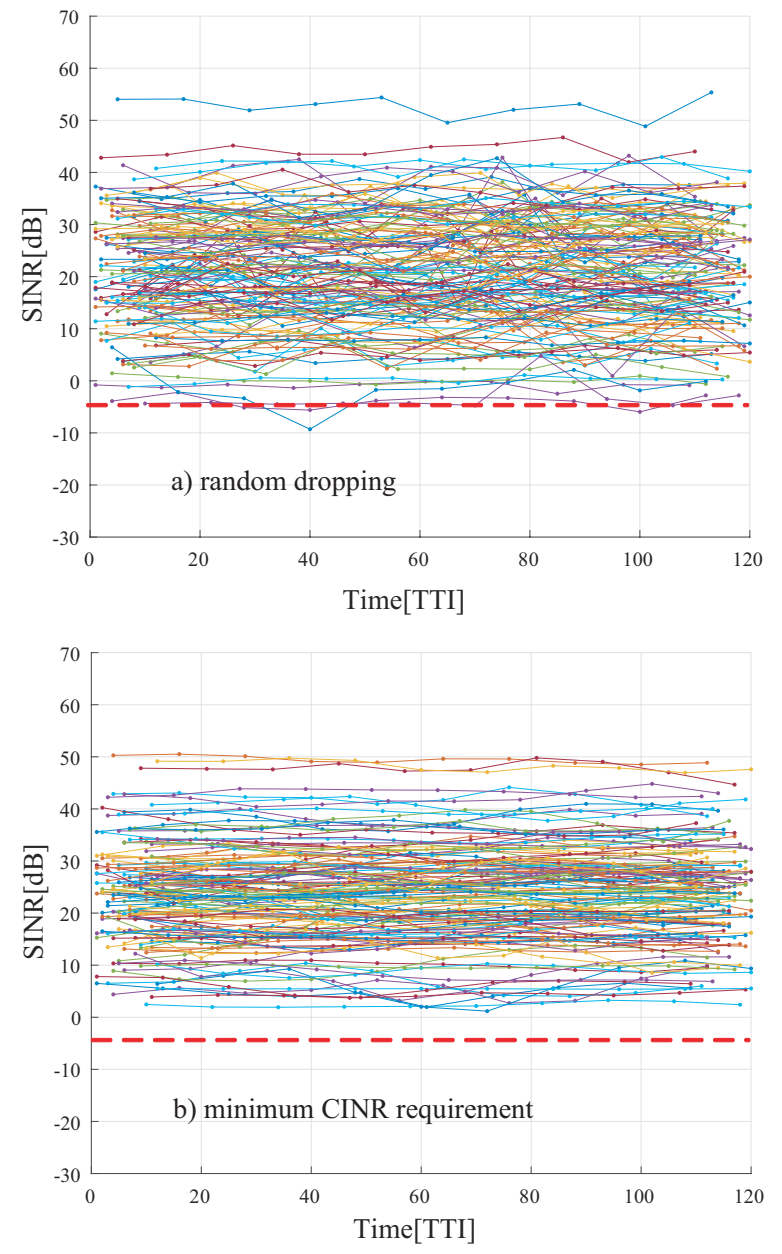

Fig. 3. Received SINR plot of Random vs CINR requirement based MS placement

MCS level, while meeting the outage probability requirement is a key to efficient network resource utilization. We consider the tail statistics [4] of the interference distribution and identify the receiver with the worst SINR (i.e., measured interference power) within a serving cell boundary to solve such optimization problem.

\section{B. Position-aided MS relocation routine for predictive RRM}

As physical boundaries of an indoor cell are defined, associated UEs shall be located within the cell coverage. UEs located near the cell boundaries usually have high interference to noise ratio (INR). In order to achieve URLLC, INR should be managed for all UEs within a cell such that the worst SINR value corresponding to a lowest MCS level should not exceed the target outage BLER (e.g., $10^{-5}$ ). To avoid this, we propose a relocation routine for associated UE by comparing received carrier to interference noise ratio (CINR) to a threshold $\beta$.

The first step to position-aided UE relocation is measuring received signal's reference signal power from various sources with the following criterion, that

$$
C I N R=R S R P_{\text {serving }} / \sum R S R P_{\text {neighbor }}+n
$$
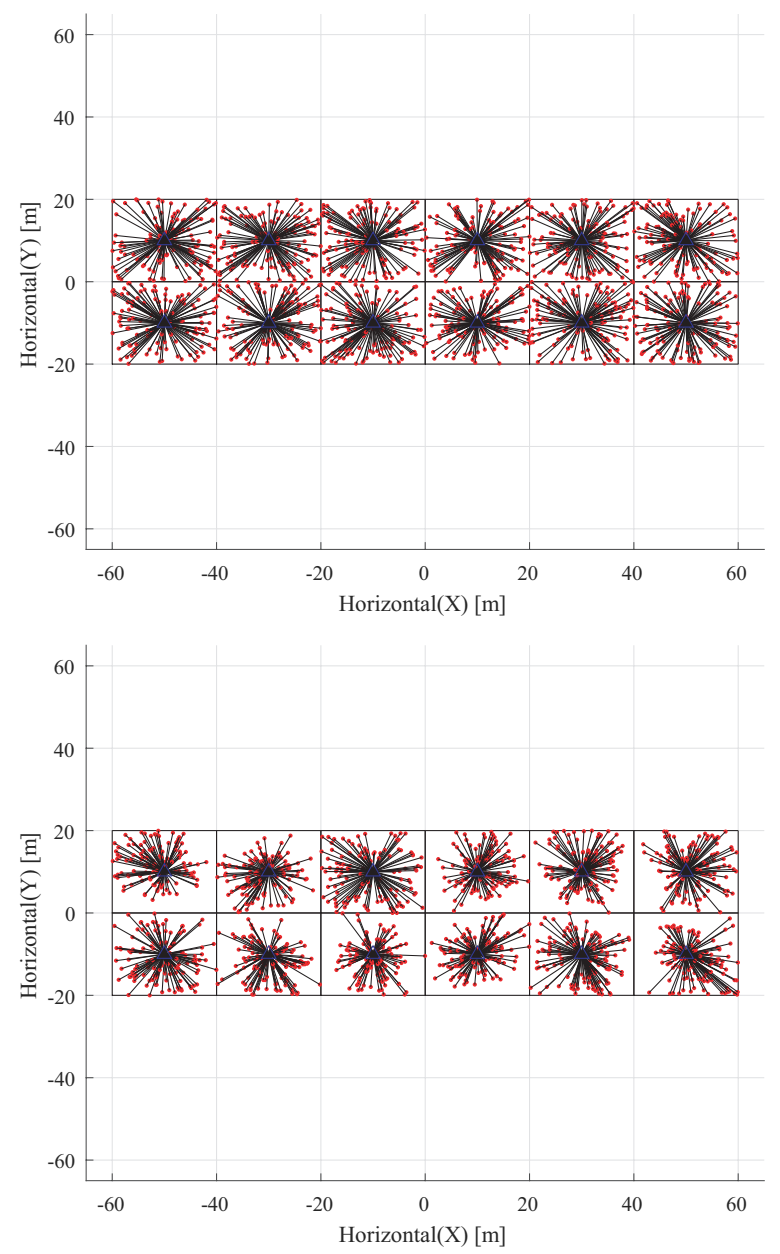

Fig. 4. MS horizontal placement results according to CINR requirements (top: $-4.88 \mathrm{~dB}$, bottom: $5 \mathrm{~dB}$ )

exceeds the minimum required SINR for MCS 0 corresponding to the target BLER (which can be obtained from the BLER curves in Fig. 2). RSRP refers to reference signal received power, and this represents the reference signal power of a serving cell versus other neighbor cells difference measured at a MS. This usually indicates the rough average SINR that can be acquired from the MS. $n$ is the additive white Gaussian noise. The BS receives SINR report from served MS to analyze the $C I N R$.

If $C I N R<\beta$, the BS can indicate that the antenna direction of the corresponding MS rotates (azimuth/zenith) toward the serving BS until the minimum $C I N R$ condition is met. If antenna angle adjustment is not effective, re-locate the MS and re-measure the $C I N R$ until it is satisfied. In the case of Table $1, \beta$ should be set to $-4.8 d B$. It is expected that the geometrical distance between the serving base station and the MSs shortens as the threshold level for CINR increases. Fig. 4 shows an experimental simulation result of MS geographical placement results after applying $C I N R$ to served MSs (120 MSs are deployed per cell in this case). As the threshold value for $C I N R$ becomes higher, it seems that MSs are placed toward the center of the serving BSs, as expected. As the 
relocation progresses, geographical location information can be saved in a database such that $C I N R$ requirements could be met by MS's positional information. This use of location information in resource management is one of the active research areas in the $6 \mathrm{G}$ [1][12]. Please note that the some MSs may not be relocated due to the indispensability of the service requirement at that location. Under such circumstances, frequency diversity or other reliability enhancement techniques can be adopted to ensure URLLC services. However, such an investigation is beyond the scope of this work.

TABLE I

SINR REQUIREMENTS TARGETED AT BLER $10^{-5}$

\begin{tabular}{c|c|c|c} 
Parameters & $\begin{array}{c}\text { Code rate } \\
\text { (QPSK) }\end{array}$ & $\begin{array}{c}\text { Req. SINR } \\
(\mathrm{dB})\end{array}$ & $\begin{array}{c}\text { Scheduled } \\
\text { UEs per mimi-slot }\end{array}$ \\
\hline MCS 0 & $\mathrm{R}=256 / 2400$ & -4.8 & 1 \\
MCS 1 & $\mathrm{R}=256 / 1200$ & -2.8 & 2 \\
MCS 2 & $\mathrm{R}=256 / 800$ & 0.06 & 3 \\
MCS 3 & $\mathrm{R}=256 / 600$ & 1.63 & 4 \\
MCS 4 & $\mathrm{R}=256 / 400$ & 4.33 & 6 \\
MCS 5 & $\mathrm{R}=256 / 400$ & 10.38 & 12 \\
& $(16 \mathrm{QAM})$ & &
\end{tabular}

Fig. 3 shows the SINR tracking result of every MS deployed in the indoor system model. It is clear that the amount of fluctuations in SINR for random MS placement within a square cell is more pronounced than the placement based on the minimum $C I N R$ requirement. In addition, the lowest measured SINR seems much lower than the MCS 0 target outage level, which leads to failure in meeting scalable URLLC reliability requirements. On the other hand, applying minimum $C I N R$ requirement seems to maintain a much narrower SINR fluctuation range. With the minimum $C I N R$ requirement, the system can setup a target outage probability and assess whether the system in physical layer perspective meets the requirements or not. For estimating the receiver's SINR, we apply the predictive RRM algorithm to choose the right MCS level and observe/compare its effectiveness in a system level simulation environment.

\section{Interference predictive RRM}

After collecting $C I N R$ reports from the served MSs within a cell, the serving cells check the link reliability requirements set by the target outage probability. This means the serving cells collect SINR reports (and/or acknowledgement) from all MSs to figure out the outage probability. Then, the serving cell schedules higher level MCS to MSs in a selected group, if the reported SINR is higher than the higher level MCS's required value. By applying the worst received SINR scheduling rule detailed in Section II A, base station can build an initially observed interference space $\mathcal{I}$. The interference space is discretized into $\mathcal{L}=\left\{\mathcal{S}_{0}, \mathcal{S}_{1}, \ldots \mathcal{S}_{L-1}\right\}$ states that covers the entire range of observed interference range measured from MSs. $L$ refers to the maximum number of quantized states. As suggested in [8], $\mathcal{I}^{2}$ value is used for equally spacing interference states since it fits well for a risk sensitive approach.
The interference transition matrix $\mathcal{P}$ that describes the transmission probability of a current interference level $\mathcal{S}_{i}$ to $\mathcal{S}_{j}$ at instance $t$ is represented by [8]

$$
\begin{aligned}
p_{i j}= & \frac{\sum_{t}\left[\mathbf{1}_{\mathcal{S}^{t+1}}\left(S_{j}\right) \mid \mathbf{1}_{\mathcal{S}^{t}}\left(\mathcal{S}_{i}\right)\right]}{\sum_{t}\left[\mathbf{1}_{\mathcal{S}^{t}}\left(S_{i}\right)\right]} \\
& \forall i, j \in\{0,1, \ldots L-1\},
\end{aligned}
$$

where $\mathbf{1}_{\mathcal{A}}(x)$ is the indicator function which equals 1 if $x \in$ $\mathcal{A}$ and 0 otherwise. For example, if $\mathcal{S}_{i}^{t}=\mathcal{S}_{j}^{t+1}$ occurs, the reported interference level from $t$ to $t+1$ is unchanged.

After building the probability transition matrix, which characterizes the interference distribution, the base station decides the downlink MCS level for the MS with the worst received SINR based on the interference prediction. The confidence level parameter $\eta<1$ that represents the minimum sum probability $\sum_{l=0}^{j} p_{i l} \geq \eta$ incorporates all possible transition possibilities of the discretized interference levels. This can be represented as

$$
\operatorname{Pr}\left[I^{t+1} \leq \hat{I}^{t+1}\right]>\eta,
$$

where $I^{t+1}$ refers to the actual interference and $\hat{I}^{t+1}$ refers to predicted interference at the next state.

For example, if $L=20$ and the current interference level is $i=3$, while $j=4$ such that the minimum interference level is greater than a $\eta$ indicates the predicted interference level. This means the predicted interference level in the next state is greater than the current level. Based on the predicted interference level $L$, the BS would look up the required SINR such as Table 1 to decide an MCS level.

\section{NUMERICAL RESULTS}

In this section, we compare the system level simulation (SLS) results with predictive RRM based MCS scheduling rule and the baseline conventional SINR moving average based MCS scheduling method [13]. The baseline moving average estimator receives interference report from MS and filters the reported interference value through a low-pass IIR filter. The predicted interference estimate would be

$$
\hat{I}^{t+1}=\alpha I^{t-1}+(1-\alpha) \hat{I}^{t},
$$

where $\alpha$ is a weight factor.

The evaluation assumptions and parameters are given as follows: $12 \mathrm{MS}$ per cell are dropped in the cell layout, center frequency of $3.5 \mathrm{GHz}$ is applied, $60 \mathrm{kHz}$ subcarrier spacing, mini-slot of 4 OFDM symbols $(0.071 \mathrm{~ms}$ of transmission time interval (TTI)), 20MHz system bandwidth, 50 REs assigned, 32 bytes of payload size, and up to $12 \mathrm{MS}$ served per BS in every mini-slot. The indoor office has 12 BSs and all nodes are assumed to operate independently. Each BS is equipped with $4 \times 4 \times 2$ (VxHxPol) antenna panels with 20 meter intersite distance. A single ( $\mathrm{VxHxPol})$ antenna panel is applied for all MSs.

The performance of the proposed position-aided MS relocation and interference predictive RRM method is numerically evaluated against the conventional moving average based 

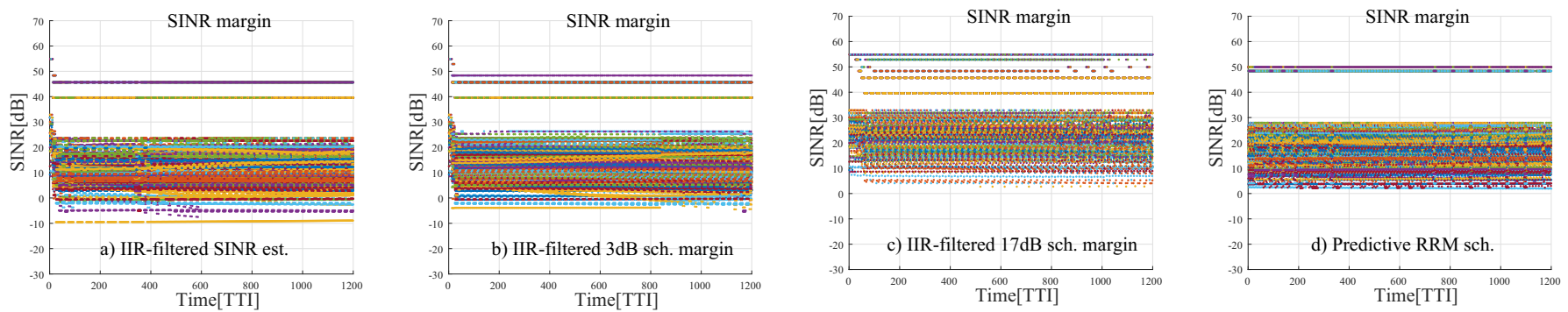

Fig. 5. SINR margin of baseline IIR-filtered vs proposed predictive RRM

SINR estimation scheme. For the baseline, we assume that relocation of MS is not applied, once MSs are dropped in cell boundaries. The interference state $L$ is divided into 20 states. For the proposed scheme, risk sensitivity value indicated in Eq. (3) is set to $\eta=0.95$.

1) SINR margin: We first evaluate the SINR margin of two schemes as a function of scheduled TTI. The SINR margin refers to a gap between received downlink signal's SINR and the required SINR of a scheduled MCS at a target BLER. Thus, a positive SINR margin refers to a successful transmission status. On the other hand, if the margin is way too much a positive value, it indicates that resource is used inefficiently. Fig. 5 shows SINR margins of all MSs served by the $12 \mathrm{BSs}$. It can be clearly identified that SINR margin of the proposed scheme (Fig. 5d) shows all positive values during the time interval of 120 TTI, whereas the baseline IIRfiltered feedback SINR estimation scheme (Fig. 5a) displays quite a few large negative values in the SINR margin vs TTI plot. We also applied $3 \mathrm{~dB}$ and $17 \mathrm{~dB}$ scheduling margin to observe further detail on SINR margin statuses. A typical $3 \mathrm{~dB}$ scheduling margin is selected to decide whether negative SINR margin improves or not, and $17 \mathrm{~dB}$ scheduling margin is chosen such that negative SINR margin would not occur. The performance of baseline IIR-filtered SINR estimation scheme does not meet the reliability requirement as SINR margin tracking records in Fig. 5a and 5b show some negative values. However, a high SINR margin of $17 \mathrm{~dB}$ (Fig. 5c) would be demanded to make the system overcome the URLLC constraints, but at the expense of higher resource usage.

2) Average transmit packets per TTI: Next, we evaluate the average transmitted packet of the compared scheduling methods. Table II shows a summarized resource efficiency in terms of the average number of transmitted slots (not accounting NACK nor retransmission) per TTI. The fixed MCS 0 is a special case of a scheduling rule that all BSs do not apply any rate adaptation scheme. This mode is set to initially analyze the lower bound of system outage probability, and subsequent MS relocations would help achieve the target bound. For the current indoor SLS environments and parameter set, MCS 0 only transmission mode with MS relocation meeting CINR requirement leads to zero transmission error.

Baseline IIR-filtered scheduling mode has three different configurations, as shown in Table II. With OdB SINR margin, average transmission rate is reached up to 8.553 slots per TTI, whereas either $3 \mathrm{~dB}$ or $17 \mathrm{~dB}$ scheduling margin reduces the network downlink throughput. The $17 \mathrm{~dB}$ scheduling margin case would make the system achieve the reliability target, but the resource efficiency drops below the predictive interference estimation based RRM scheduling method.

TABLE II

AVERAGE TRANSMIT PACKETS PER TTI

\begin{tabular}{c|c|c|c}
$\begin{array}{c}\text { Scheduling } \\
\text { margin }\end{array}$ & $\begin{array}{c}\text { fixed } \\
\text { MCS } 0\end{array}$ & $\begin{array}{c}\text { Baseline IIR-filtered } \\
\text { est. SINR }\end{array}$ & Predictive RRM \\
\hline 0dB & 1.00 & 8.553 (sys. outage) & 3.318 \\
3dB & - & 7.333 (sys. outage) & - \\
17dB & - & 1.854 & -
\end{tabular}

3) Theoretical comparison of resource allocation in SLS interference environment from fixed MCS mode scheduling: We compare the outage performance and resource usage of the comparing schemes. The received downlink samples were collected from the SLS with CINR requirement based relocation procedure and fixed MCS 0 mode scheduling. Since fixed MCS 0 transmission already achieves the reliability constraint of BLER $10^{-5}$, we can analyze the expected actual outage probability and resource usage from the given interference environment. The theoretical block error rate $\epsilon$ and resource usage $R$ can be evaluated from [14][15]

$$
R \approx \frac{D}{C(\gamma)}+\frac{Q^{-1}(\epsilon)^{2} V(\gamma)}{2 C(\gamma)^{2}}\left[1+\sqrt{1+\frac{4 D C(\gamma)}{Q^{-1}(\epsilon)^{2} V(\gamma)}}\right]
$$

where $\gamma=S N R / \hat{I}$ is the predicted SINR. $C(\gamma)=\log _{2}(1+\gamma)$ is the Shannon capacity of AWGN channels under infinite blocklength regime. $Q^{-1}(\cdot)$ is the inverse of the Qfunction. $V(\gamma)=\frac{1}{\ln (2)^{2}}\left(1-\frac{1}{(1+\gamma)^{2}}\right)$ is the channel dispersion. To achieve a low outage probability, cost of resource usage increases. The usage of resource and target versus actual/achievable outage performance is shown in Fig. 6. As expected, the predictive interference based RRM requires more resource than the ideal case of transmission rate/capacity. However, the proposed scheme in [8] is analyzed to have higher resource efficiency with higher reliability than the baseline scheme. This translates to predictive RRM based rate adjustment method being much more accurate in terms of estimating future SINR values. 

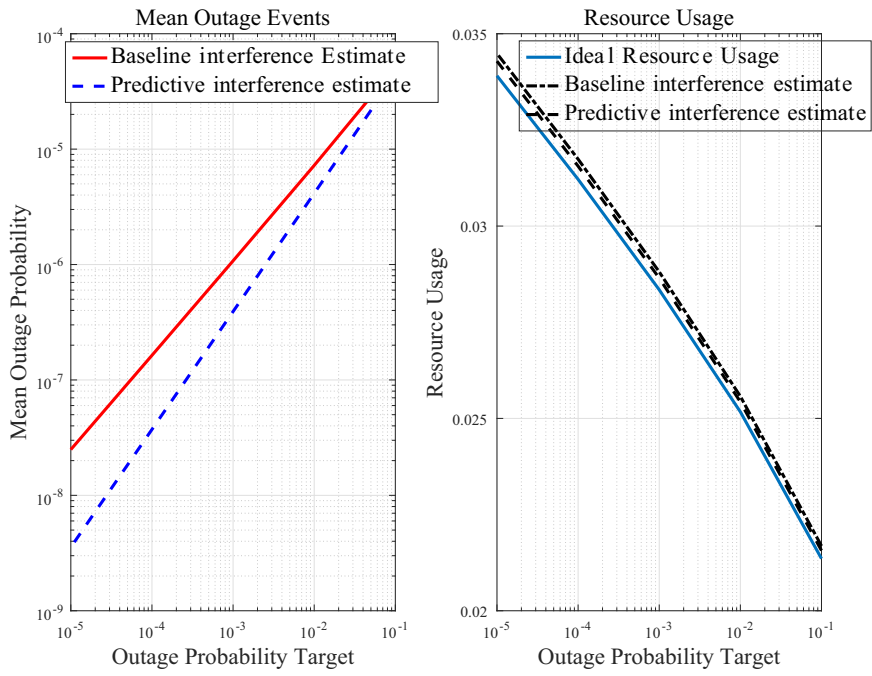

Fig. 6. Fixed MCS 0 scheduling based data sample comparison of mean outage and resource usage

\section{CONCLUSiON}

In this paper, we proposed a novel location aware transmission rate adjustment scheduling method for URLLC networks based on the predictive interference management algorithm. Our suggestion to re-locate some receivers in order to fulfill target outage probability first ensures reliability and latency requirements. The risk sensitive predictive interference RRM is proven to significantly enhance both reliability and resource efficiency in a 3D system level, compared to conventional IIRfilter based average SINR estimation based scheduling scheme.

\section{ACKNOWLEDGMENT}

This work was supported by Institute for Information \& communications Technology Promotion (IITP) grant funded by the Korea government (MSIT) (No. 2020-0-01316, International cooperation and collaborative research on $5 \mathrm{G}+$ technologies for ultra-reliability low latency communications and the Academy of Finland 6Genesis Flagship program (grant no. 318927).

\section{REFERENCES}

[1] N. H. Mahmood et al. "White paper on critical and massive machine type communication towards (6G)," University of Oulu, June 2020. http://jultika.oulu.fi/files/isbn9789526226781.pdf, pp. 1-36.

[2] METIS, Deliverable D1.1 Scenarios, requirements and KPIs for 5G mobile and wireless systems, May 2013.

[3] B. Holfed et al, "Wireless communication for factory automation: an opportunity for LTE and 5G systems," IEEE Commun. Mag., vol. 54, no. 6, pp. 361-43, June 2016.

[4] M. Bennis, M. Debbah, and H. V. Poor, "Ultrareliable and low-latency wireless communication: Tail, risk, and scale," Proc. of IEEE, vol. 106, no. 10, pp. 1834-1853, Oct. 2018.

[5] N. A. Johansson, Y.-P. E. Wang, E. Eriksson, M. Hessler, "Radio access for ultra-reliable and low-latency $5 \mathrm{G}$ communications," IEEE International Conference on Communications (ICC) Workshop on $5 G \&$ Beyond - Enabling Technologies and Applications, June 2015.

[6] J. Sachs and L. A. A. Andersson and J. Araújo and C. Curescu and J. Lundsjö and G. Rune and E. Steinbach and G. Wikström, "Adaptive 5G low-latency communication for tactile internet services," Proc. of IEEE, vol. 107, no. 2, pp. 325-349, Feb. 2019.
[7] A. Wolf and P. Schulz and M. Dörpinghaus and J. C. S. Santos Filho and G. Fettweis, "How reliable and Capable is multi-connectivity?" IEEE Trans. Commun., vol. 67, no. 2, pp. 1506-1520, Feb. 2019.

[8] N. H. Mahmood, O. A. Lopez, H. Alves, and M. Latva-Aho, "A predictive interference management algorithm for URLLC in beyond $5 \mathrm{G}$ networks," IEEE Commun. Lett., vol. 25, no. 3, pp. 995-999, Mar. 2021.

[9] M. Li, C. Chen, C. Hua, and X. Guan, "A learning-based pre-allocation scheme for low-latency access in industrial wireless networks," IEEE Trans. Wireless Commun., vol. 19, no. 1, pp. 650-664, Jan. 2020.

[10] N. Brahmi, O. N. C. Yilmaz, K. Wang. Helmersson, S. A. Asharf, and J. Torsner, "Deployment strategies for ultra-reliable and lowlatency communication in factory automation," Proc. of IEEE Globecom Workshops, Dec. 2015.

[11] 3GPP TSG RAN, "Study on channel model for frequencies from 0 to $100 \mathrm{GHz}$ (Release 15)," 3GPP TR 38.901, V15.0.0, June 2018.

[12] O. Kanhere and T. S. Rappaport, "Position location for futuristic cellular communications - 5G and beyond," IEEE Commun. Magazine, vol. 59, pp. 70-75, Jan. 2021.

[13] G. Pocovi, B. Soret, K. I. Pedersen, and P. Mogensen, "MAC layer enhancements for ultra-reliable low-latency communications in cellular networks," Proc. of IEEE Int. Conf. Commun. (ICC) Workshops, pp. 1005-1010, May. 2017.

[14] Y. Polyanskiy, H. V. Poor, and S. Verdu, "Channel coding rate in the finite blocklength regime," in IEEE Trans. Inf. Theory, vol. 56, no. 5, pp. 2307-2359, May 2010.

[15] A. Anand and G. de Veciana, "Resource allocation and HARQ optimization for URLLC traffic in 5G wireless networks," in IEEE J. Sel. Areas Commun., vol. 36, no. 11, pp. 2411-2421, Nov. 2018. 